\title{
REPERTOIRE
}

\section{DES ESPÈCES ET DES GENRES NOUVEAUX}

\section{Incertæ sedis}

Eatopolypoides Martin Mayer. Espèce type : E. macaci Martin Mayer. Archiv f. Schiffs- und Tropenhygiene, XXXVII, 1933, p. 504.

Entopolypoides macaci Martin Mayer. Sang. Macacus cynomolgus (Mamm). Java. Archiv f. Schiffs- und Tropenhygiene, XXXVII, 1933, p. 504.

Rickettsia blanci Caminopetros, Pheloukis et Contos. Fièvre boutonneuse du cheval. Grèce. Bull. Acad. méd. Paris, CIX, 1933, p. 835.

Rickettsia conori Brumpt. Cavilé générale. Rhipicephalus sanguineus, fièvre boutonneuse. C. R. Soc. biol., CX, 1932, p. 1199.

M. LANGERoN.

\section{Spirochète}

Spirochaeta turicatae E. Brumpt. Spirochælide. Ornilhodorus turicata (Arachn.). Austin, Texas. C. R. Soc. biol., CXIII, 1933, p. 1369.

M. L.

\section{Rhizopode}

Entamœba phallusiae Mackinnon et Ray. Amobidæ. Intestin. Phallusia mamillata (Tunic.). Plymouth. Journ. of marine biol. assoc. Unit. Kingdom, XVII, 1931, p. 585.

M. L.

\section{Sporozoaires}

Eimeria gobii Fantham. Eimeridæ. Intestin. Gobius nudicers (Poiss.). St-James près du Cap. South african journ. of sc., XXIX, 1932, p. 635.

Isospora naiae Fantham. Eimeridæ. Intestin. Naia flava (Rept.). Province occidentale du Cap. South african journ. of sc., XXIX, 1932, p. 636.

Henneguya travassosi Guimaraes et Bergamin. Myxosporidie. Poissons d'eau douce. Cruzeiro, Etat de São Paulo, Brésil. Rev. de biol. e hyg., IV, 1933, p. 41.

Isospora henryi Yakimoff et Matikaschwili. Eimeridre. Intestin. Bubo bubo (Ois.). Russie. Bull. Soc. pathol. exol., XXV, 1932, p. 1046.

Babesia motasi Wenyon. Babesiidæ. Sang. Mouton. Roumanie. Wenyon, Protozoology, II, 1926, p. 1006.

Annales de Parastrologie, T. XII, $x^{\circ} 4 .-1^{\text {er }}$ juillet 1934 , p. 332-336, 
Babesia serganti Wenyon. Babesiida. Sang. Mouton. Algérie. Wenyon, Prolozoology, II, 1926, p. 1007.

Leucocytogregarina bovis (Martoglio et Carpano) F. de Gasperi. Hepalozoidx. Rate et sang. Bœuf. Italie. Nom. nov. pro Hrmogregarina bovis Martoglio et Carpano. Riv. di malariol., XII, 1933, p. 366.

Eimeria pintoensis F, da Fonseca. Eimeridx. Intestin. Sylvilagus minensis (Mamm.). Butantan, Etat de São Paulo, Brésil. Mem. Inst. Bulantan, VII, 1932 , p. 175.

Haemogregarina butantanensis Arantes. Hæmogregarinidæ. Sang. Ophis merremii Wagler 1824 (Rept.). Brésil. Mem. Inst. Butantan, VI, 1931, p. 237.

Meroselenidium keilini Mackinnon et Ray. Gregarinida. Intestin. Polamilla reniformis (Annél.). Plymouth. Parasilology, XXV, 1933, p. 146.

Selenidium potamillae Mackinnon et Ray. Gregarinidæ. Intestin. Polamilla reniformis (Annél.). Plymouth. Parasilology, XXV, 1933, p. 153.

Eimera pigra Léger et Bory. Eimeridæ. Intestin. Scardinius erylhrophthalmus (Poiss.). France. C. R. Acad. sc., CXCIV, 1932, p. 1710.

Metchnikovella polydorae Reichenow. Melchnikovellida, Dans un Selenidium (cruzi ?) (Sporoz.) de Polydora (Annél.). Helgoland. Die Tierwell der Nord- und Oslsee, II, g, Sporozoa, 1932, p. 21.

Amphiamblys ancorae Reichenow. Melchnikovellidr. Dans Ancora sagittala (Sporoz.) de Capilella capilala (Annél.). Helgoland. Die Tierwelt der Nord-und Oslsee, II, g, Sporozoa, 1932, p. 21.

Selenidium intraepitheliale Reichenow. Gregarinidæ. Intestin. Scolelepis fuliginosa (Annél.). Helgoland. Die Tierwell der Nord- und Ostsee, II, g, Sporozoa, 1932, p. 24.

Eimeria botelhoi Carini. Eimeridæ. Intestin. Guerlinguetus ingrami (Mamm.). Brésil, Santos. Rev. de biol. e hyg. São Paulo, III, 1932, p. 80.

Myxidium lindoyense Carini. Myxosporidie. Vésicule biliaire. Bufo marinus, H Hla rubra, H. nebulosa, Leplodaclylus ocellalus, Paludicula signifera (Batr.). Lindoya, Brésil. Rev. de biol. e hyg. São Paulo, III, 1932, p. 83.

Caryospora brasiliensis Carini. Eimeridæ. Intestin. Chlorosoma astivum (Rept.). São Paulo, Brésil. Rev. de biol. e hyg. São Paulo, III, 1932, p. 85.

Eimeria bitis Fantham. Eimerida. Vésicule biliaire. Bitis arietans (Rept.). Le Cap. South African journ. of sc., XXIX, 1932, p. 630.

Eimeria clini Fantham. Eimeridr. Intestin. Clinus superciliosus (Poiss.). St-James près du Cap. South african journ. of sc., XXIX, 1932, p. 633.

Eimeria nans Yakimoff. Eimeridr. Intestin. Capra sibirica ; Ovis musimon, O. orientalis (Mamm.). Ukraine (Russie). Bull. Soc. pathol. exot., XXVI, 1933, p. 1195.

Plasmodium knowlesi Sinton et Mulligan. Plasmodidx. Sang. Silenus irus (Mamm.). Malaisie. Rec. malaria survey of India, III, 1932, p. 379.

Steinina metaplaxi Pearse. Gregarinida. Intestin. Metaplax dentipes (Crust.). Port Canning, Matla River (delta du Gange). Rec. Indian Mus., XXXIV, 1932, p. 293.

Isospora arctopitheci Rodhain. Eimeride. Intestin. Hapale jacchus penicillalus (Mamm.) (Ouistiti) mort à Anvers. C. R. Soc. biol., CXIV, 1933, p. 1357. 
Haemogregarina serini H. de Beaurepaire Aragão. Hæmogregarinidæ. Sang. Serinus canarius. (Ois.). Rio de Janeiro. C. R. Soc. biol., CXIII, 1933, p. 214.

Nosema herpobdellae Conet. Microsporidie. Tissu conjonctif. Herpobdella (Nephelis) octoculata (Annél.) Louvain (Belgique). Ann. Soc. scient. Bruxelles, LI, B. C. R. des séances, 3e section, 1931, p. 170.

M. L.

\section{Trématode}

Haplorchis milvi N. Gohar. Haplorchidæ. Intestin grêle. Milvus migrans (Ois.). Egypte. Ann. Parasitol., XII, 1934, p. 223.

M. Neveu- Lemaire.

\section{Acanthocéphales}

Nephridiacanthus manisensis A. Meyer. Oligacanthorhynchidæ. Fèces. Manis tricuspis (Mamm.). Lolodorf (Cameroun). Zool. Jahrb. Syst., LXII, 1931, p. 80 .

Moliniformis kalahariensis A. Meyer. Moniliformidæ. Intestin. Erinaceus frontalis (Mamm.), Kooa (Kalahari) et Plerocles namaqua (Mamm.), Moorane (Afrique). Zool. Jahrb. Syst., LXII, 1931, p. 82.

Pachysentis A. Meyer. Pachysentidæ. Espèce type : P. ehrenbergi A. Meyer Zool. Jahrb. Syst., LXII, 1931, p. 86.

Pachysentis ehrenbergi A. Meyer. Pachynsentidæ. Canis vulpecula (Mamm.). Egypte. Zool. Jahrb. Syst., LXII, 1931, p. 87.

Pachysentis canicola A. Meyer. Pachysenlidæ. Canis sp. (Mamm.). Brésil. Zool. Jahrb. Syst., LXII, 1931, p. 89.

Pachysentis procumbens A. Meyer. Pachysentidæ. Canis vulpecula et C. megalotis (vraisemblablement Megalolis zerda) (Mamm.). Egypte. Zool. Jahrb. Syst., LXII, 1931, p. 92.

Oncicola dimorpha A. Meyer. Pachysenlidæ. Intestin. Felis pardus (Mamm.). Duala (Cameroun). Zool. Jahrb. Syst., LXII, 1931, p. 97.

Oncicola bursata A. Meyer. Pachysentidæ. Intestin. Felis moormensis (Mamm.). Malacca. Zool. Jahrb. Syst., LXII, 1931, p. 99

Oncicola gigas A. Meyer. Pachysentidæ. Felis pardus melas (Mamm.). Lccalité non indiquée. Zool. Jahrb. Syst., LXII, 1931, p. 100.

Oncicola macrurae A. Meyer. Pachysentida. Felis macrura (Mamm.). Conception (Paraguay). Zool. Jahrb. Syst., LXII, 1931, p. 101.

Echinopardalis macrurae A. Meyer. Pachysentidæ. Felis macrura (Mamm.). Amérique du sud. Zool. Jahrb. Syst., LXII 1931, p. 103.

Echinopardalis atrata A. Meyer. Pachysentidx. Herpestes ichneumon, Felis catus domesticus, Canis valpecula (Mamm.). Egypte. Zool. Jahrb. Syst., LXII, 1931 , p. 105.

Echinopardalis decrescens A. Meyer. Pachysentidæ. Intestin. Felis macrura, localité non indiquée ; Felis sp., Brésil (Mamm.). Zool. Zahrb. Syst., LXII, 1931, p. 107. 
Centrorhynchus maryasis M. N. Data. Centrorhynchida. Intestin. Uracissa melanocephala occipitalis '(Ois.). Inde. Ind. Science Congress. 18th. ann. meet. Abst., sect. zool., 1931, p. 3.

Centrorhynchus reptans G. D. Bhalerao. Centrorhynchidx. Naja hannah (Rept.). Muktesar (Inde). Ind. Science Congress, 18th. ann. meet. Abst. sect. zool., 1931, p. 3 .

\section{R.-Ph. Dollfus .}

\section{Copépode}

Ergasilus japonicus I. Harada. Ergasilida. Membrane muqueuse de la base des nageoires et surface de l'opercule. Cultriculus kneri, Pseudorasbora parva, Pararasbora moltrechli, lac Jitsugetsutan (Formose); Zacco temmincki Tensonpi Rato (Formose), (Poiss.). Jl. Soc. trop. Agric. Japan, II, 1930, p. 71 .

$$
\text { R. -Ph. D. }
$$

\section{Isopode}

Ichthyoxenus formosanus I. Harada. Cymothoidæ. Sac membraneux situé ventralement et en arrière des nageoires pectorales. Carassius auratus (Po'ss.). Lac Jitsugetsutan (Formose). Jl. Soc. trop. Agric. Japan, II, 1930, p. 265.

$$
\text { R.-PH. D. }
$$

\section{Acariens}

Trombicula trifurca H. E. Ewing. Trombidiidæ. Larve parasite probablement sur Chéiroptères (Mamm.). Chilibrillo Caves (Panama). Proc. U. S. Nat. Mus., no 2970, LXXXII, 1933, p. 3.

Trombicula cavernarum H. E. Ewing. Trombidiidæ. Hôte inconnu. Chilibrillo Caves (Panama). Proc. U.S. Nat. Mus., no 2970, LXXXII, 1933, p. 4.

Trombicula hominis H. E. Ewing. Trombidiidx. Peau. Enfant. Aguabuenas (Panama) Proc. U. S. Nat. Mus., no 2970, LXXXII, 1933, p. 5.

Acanthochela H. E. Ewing. Parasitida. Espèce type : A. chilensis H. E. Ewing. Proc. U.S. Nat Mus., no 2971, LXXXII, 1933, p. 1.

Acanthochela chilensis H. E. Ewing. Parasitidx. Peau. Didelphis elegans (Mamm.). Lota (Chili). Proc. U. S. Nat. Mus., no 2971, LXXXII, 1933, p. 2.

Haemogamasus sternalis H. E. Ewing. Parasitidx. Peau. Blarina brevicauda talpoides (Mamm.). Adirondack Lodge, Essex county (N. Y., U. S. A.). Proc. U. S. Nat. Mus., no 2971, LXXXII, 1933, p. 3.

Euhaemogamasus H. E. Ewing. Parasilidæ. Espèce type : E. onychomydis H. E. Ewing. Proc. U.S. Nal. Mus., no 2971, LXXXII, 1933, p. 3.

Euhaemogamasus onychomydis H. E. Ewing. Parasitidæ. Peau. Onychomys sp. (Mamm.). Oraibi (Arizona). Proc. U. S. Nat. Mus., n ${ }^{\circ}$ 2971, LXXXII, 1933, p. 4 .

Euhaemogamasus utahensis H. E. Ewing. Parasilida. Peau. Neotoma lepida lepida (Mamm.). Salina (Utah). Proc. U. S. Nal. Mus.; no 2971. LXXXII, 1933, p. 4. 
Euhaemogamasus oregonensis H. E. Ewing. Parasitidæ. Peau. Phenacomys albipes (Mamm.). Netarts (Orégon). Proc. U. S. Nat. Mus., nº 2971, LXXXII, 1933, p. 5.

Cyclolaelaps H. E. Ewing. Parasitida. Espèce type: C. circularis H. E. Ewing. Proc. U. S. Nat. Mus., no 2971, LXXXII, 1933, p. 5.

Cyclolaelaps circularis H. E. Ewing. Parasilidæ. Peau. Peromyscus truei truei (Mamm.). Salina (Utah). Proc. U.S. Nal. Mus., no2971, LXXXII, 1933, p. 6.

Macrolaelaps peruvianus H. E. Ewing. Parasitidæ. Peau. "Wildrat " (Mamm.). Lima (Pérou). Proc. U. S. Nat. Mus., no 2971, LXXXII, 1933, p. 7.

Hemilaelaps H. E. Ewing. Parasitidæ. Espèce type : H. americanus H. E. Ewing. Proc. U. S. Nal. Mus., no 2971, LXXXII, 1933, p. 7.

Hemilaelaps americanus H. E. Ewing. Parasilidx. Peau. Drymarchon corais melanurus (Rept.). Brownsville (Texas). Proc. U. S. Nat. Mus., n² 2971, LXXXII, 1933, p. 8.

Hemilaelaps distinctus H. E. Ewing. Parasilida. Peau. Elaphe obsolela (Rept.). Kentrecky. Proc. U. S. Nat. Mus., no 2971, LXXXII, 1933, p. 8.

Tetragonyssus microti H. E. Ewing. Dermanyssidæ. Peau. Microlus californicus Los Angeles (Californie) et Microtus sp., Flat (Alaska.).(Mamm.). Proc. U. S. Nal. Mus., n ${ }^{\circ}$ 2971, LXXXII, 1933, p. 9.

Ceratonyssus occidentalis H. E. Ewing. Dermanyssidæ. Peau. Myotis (?) sp. "common brownbat" (Mamm.). Adel (Oregon). Proc. U. S. Nat. Mus., no 2971 , LXXXII, 1933, p. 10 . .

Dermanyssus evotomydis H. E. Ewing. Dermanyssidæ. Peau. Evolomys sp. (Mamm.). Mount Me Intyre (New-York). Proc. U. S. Nat. Mus., no 2971, LXXXII, 1933, p. 11.

Dermanyssus prognephilus H. E. Ewing. Dermanyssidw. Peau. Progne subis (Ois.). Bell (Maryland). Proc U. S. Nal. Mus., no 2971, LXXXII, 1933, p. 12.

Sommatericola orientalis H. E. Ewing. Dermanyssidæ. Peau. Lanius nigriceps longicandalus (Ois.). Bangkok (Siam). Proc. U. S. Nat. Mus., no 2971, LXXXII, 1933, p. 13.

Cyclacarus H. E. Ewing. Uropodida. Espèce type : C. aberrans H. E. Ewing. Proc. U. S. Nat. Mus., no 2971, LXXXII, 1933, p. 13.

Cyclacarus aberrans H. E. Ewing. Uropodidx. Plumes de la queue. Chrlura pelagica (Ois.). West Chester (Pa., U. S.A.). Proc. U.S. Nat. Mus., no 2971, LXXXII, 1933, p. 14.

$$
\text { R.-Ph. D. }
$$

\section{Diptère}

Phlebotomus almazani H. Galliard. Psychodidre. Yucatan. Ann. Parasitol., XII, 1934, p. 193.

M. Neveu-Lemaire.

Le Gérant: F. Amirault. 